\title{
Numerical simulations of concrete flow: A benchmark comparison
}

Roussel, Nicolas; Gram, Annika; Cremonesi, Massimiliano; Ferrara, Liberato; Krenzer, Knut; Mechtcherine, Viktor; Shyshko, Sergiy; Skocec, Jan; Spangenberg, Jon; Svec, Oldrich Total number of authors: 12

Published in:

Cement and Concrete Research

Link to article, DOI:

10.1016/j.cemconres.2015.09.022

Publication date:

2016

Document Version

Peer reviewed version

Link back to DTU Orbit

Citation (APA):

Roussel, N., Gram, A., Cremonesi, M., Ferrara, L., Krenzer, K., Mechtcherine, V., Shyshko, S., Skocec, J., Spangenberg, J., Svec, O., Thrane, L. N., \& Vasilic, K. (2016). Numerical simulations of concrete flow: A benchmark comparison. Cement and Concrete Research, 79, 265-271. https://doi.org/10.1016/j.cemconres.2015.09.022

\section{General rights}

Copyright and moral rights for the publications made accessible in the public portal are retained by the authors and/or other copyright owners and it is a condition of accessing publications that users recognise and abide by the legal requirements associated with these rights.

- Users may download and print one copy of any publication from the public portal for the purpose of private study or research.

- You may not further distribute the material or use it for any profit-making activity or commercial gain

- You may freely distribute the URL identifying the publication in the public portal 


\section{Elsevier Editorial System(tm) for Cement and Concrete Research Manuscript Draft}

Manuscript Number:

Title: NUMERICAL SIMULATIONS OF CONCRETE FLOW: A BENCHMARK COMPARISON

Article Type: Research Paper

Keywords: Fresh Concrete (A); Rheology (A); Workability (A); Modeling (E); casting

Corresponding Author: Dr. Nicolas Roussel,

Corresponding Author's Institution: Université Paris Est, IFSTTAR

First Author: Nicolas Roussel

Order of Authors: Nicolas Roussel; Annika Gram; Massimiliano Cremonesi; Liberato Ferrara; Knut Krenzer; Viktor Mechtcherine; Sergiy Shyshko; Jan Skocec; Jon Spangenberg; Oldrich Svec; Lars N Thrane; Ksenija Vasilic

Abstract: First, we define in this paper two benchmark flows readily usable by anyone calibrating a numerical tool for concrete flow prediction. Such benchmark flows shall allow anyone to check the validity of their computational tools no matter the numerical methods and parameters they choose. Second, we compare numerical predictions for these two benchmark flows obtained by various research teams around the world using various numerical techniques. Our results show that all numerical techniques compared here give very similar results suggesting that numerical simulations of concrete flow have reached a technology readiness level allowing them to move from the lab to the industrial practice.

Suggested Reviewers: Frédéric Dufour

Laboratoire 3S-R

frederic.dufour@grenoble-inp.fr

Expert in numerical simulations

Dimitri Feys

Missoury University

feysd@mst.edu

Expert in concrete rheology and rheometry

Peter Bilberg

Strangbetong

peter.billberg@strangbetong.se

Expert in concrete rheology and processing 


\section{NUMERICAL SIMULATIONS OF CONCRETE FLOW: A BENCHMARK}

\section{COMPARISON}

3 Nicolas Roussel $^{1}$, Annika Gram ${ }^{2}$, Massimiliano Cremonesi ${ }^{3}$, Liberato Ferrara ${ }^{3}$, Knut Krenzer ${ }^{4}$,

4 Viktor Mechtcherine ${ }^{5}$, Sergiy Shyshko ${ }^{5}$, Jan Skocec ${ }^{6}$, Jon Spangenberg ${ }^{7}$, Oldrich Svec ${ }^{7}$, Lars

$5 \quad$ Nyholm Thrane $^{8}$, Ksenija Vasilic ${ }^{9}$

6

$7 \quad{ }^{1}$ Université Paris Est, IFSTTAR, laboratoire Navier, France.

$8 \quad{ }^{2}$ Swedish Cement and Concrete Research Institute, Stockholm, Sweden.

$9{ }^{3}$ Department of Civil and Environmental Engineering, Politecnico di Milano, Italy.

$10{ }^{4}$ IAB Weimar - Institut für Angewandte Bauforschung Weimar GmbH, Germany.

$11{ }^{5}$ TU Dresden, Institute of Construction Materials, Dresden, Germany.

$12{ }^{6}$ Heidelberg Cement Technology Center GmbH, Leimen, Germany.

$13{ }^{7}$ Department of Mechanical Engineering, Technical University of Denmark, Denmark.

$14{ }^{8}$ Danish Technological Institute, Denmark.

$15{ }^{9}$ BAM, Federal Institute for Material Research and Testing, Berlin, Germany.

Highlights

We define two benchmark flows for comparison of numerical simulations for concrete flow.

We describe the three most used numerical methods for concrete flow simulations.

We compare the predictions of these methods when used by various research teams.

21 We conclude on the technological readiness level of these numerical tools.

\section{Abstract}


1 First, we define in this paper two benchmark flows readily usable by anyone calibrating a

2 numerical tool for concrete flow prediction. Such benchmark flows shall allow anyone to

3 check the validity of their computational tools no matter the numerical methods and

4 parameters they choose. Second, we compare numerical predictions for these two

5 benchmark flows obtained by various research teams around the world using various

6 numerical techniques. Our results show that all numerical techniques compared here give

7 very similar results suggesting that numerical simulations of concrete flow have reached a

8 technology readiness level allowing them to move from the lab to the industrial practice.

10 Keywords

11 Fresh Concrete (A) ; Rheology (A) ; Workability (A) ; Modeling (E) ; casting

13 I. Introduction

14 The first use of numerical simulations of concrete flow by Mori and Tanigawa traces back to

151992 [1]. Since then, the increasing use of Self Compacting Concrete (SCC) and the growing 16 interest for rheology and processing has led to a very strong increase in the academic 17 activity in this field along with an increasing number of publications dealing with concrete 18 flow simulations using various numerical techniques. This topic is of a complex nature as it 19 involves the non-steady free surface flow of a non-Newtonian fluid. Therefore, it requires 20 the knowledge of modern computational techniques, non-Newtonian fluid mechanics and 21 the specific concrete casting processes used in civil engineering. A lot of progresses have 22 however been made in recent years. They are gathered in a recent RILEM State of the Art 23 report [2]. 
1 Despite this academic activity, the use of concrete flow simulations in industrial practice is

2 still sporadic. It can of course be expected that no one should consider simulating the casting

3 of a residential concrete slab. It is however surprising that the optimization of pre-cast

4 factory processes or the possibility to numerically forecast a critical phase of a concrete

5 construction process in the case of advanced super-structures has not drawn much attention

6 from the industry yet. We can note however that the use of these tools seem to have been

7 steadily increasing in the field of litigation as they allow, in many case, to distinguish

8 between the responsibility of the contractor and the responsibility of the concrete supplier.

9 It is our belief that the use of these advanced engineering tools is hampered by their

10 diversities and the fact that, as for all computational tools, one always get a result but,

11 without experience, one has no clue about the meaningfulness of the obtained prediction.

12 This paper focuses therefore on demonstrating that numerical simulations of concrete flow

13 are now fully able to predict accurately concrete flows are now a mature technology fully

14 able to accurately predict concrete flows and that these scientific and engineering tools are now ready to be used for a wide range of either academic or industrial purposes.

16 Our objectives here are two-fold. First, we define two benchmark flows readily usable by 17 anyone calibrating a numerical tool for concrete flow prediction. Such benchmark flows shall 18 allow anyone to check the validity of their computational tools no matter which numerical 19 methods and parameters are chosen. Second, we compare numerical predictions for these 20 two benchmark flows obtained by various research teams around the world using various 21 numerical techniques. Our results suggest that numerical simulations of concrete flow have 22 reached a technology readiness level allowing them to move from the lab to the industrial 23 practice. 


\section{The studied benchmark flows}

3

4 We choose in this work to study and compare numerical predictions for two benchmark

5 flows, namely the slump flow and the channel flow. These tests were chosen because

6 approximate analytical solutions for the final shape of the concrete sample do exist. It can

7 moreover be kept in mind that two benchmark flows are necessary, as all numerical

8 methods compared here are not using the same input parameters. For instance, whereas

9 Computational Fluid Dynamics methods (CFD) are using standard rheological parameters

10 such as yield stress and plastic viscosity as input, Distinct Element Methods (DEM) are using

11 interaction parameters between constitutive particles chosen to mimic the behavior of a

12 given concrete (Cf. section 3 ). We use therefore in this paper the slump flow as a calibrating

13 benchmark flow for all methods while we compare the numerical predictions in the case of

14 the channel flow.

\subsection{The virtual concrete}

The virtual concrete, which is studied here and implemented in the codes of the various CFD tools that are compared, can be described by a Bingham model. It has a yield stress of $50 \mathrm{~Pa}$,

20 a plastic viscosity of 50 Pa.s and a density of $2300 \mathrm{~kg} / \mathrm{m}^{3}$. This concrete is virtually mimicked by the DEMs by tuning the particle interaction laws until the behavior of the resulting virtual material is similar to the one of a $50 \mathrm{~Pa}$ yield stress material with a plastic viscosity of 50 Pa.s. 
$14 \quad h(r)=\left(\frac{2 \pi_{c}\left(h^{\prime}-r\right)}{p !}\right)^{\frac{1}{2}}$

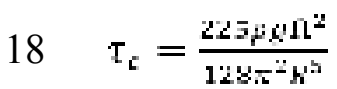

\subsection{The slump flow}

$$
\tau_{c}=\frac{223 \dot{s} g \Lambda^{2}}{128 \pi^{2} h^{2}}
$$

\subsection{The channel flow}

We are considering here the standard Abrams cone geometry, which corresponds to $\mathrm{H}_{0}=$ $300 \mathrm{~mm}, R_{\min }=50 \mathrm{~mm}$ and $R_{\max }=100 \mathrm{~mm}$ using the notations in Fig. 1.

Roussel and Coussot [3] described the various relations existing between slump/slump flow and yield stress from an analytical point of view for two asymptotic situations, namely $H \gg R$ and $H \ll R$ ( $H$ and $R$ being the height and radius of the sample, respectively). In these two situations, the governing flow equations can be significantly simplified in order to obtain approximate analytical solutions. We focus here on the case $H \ll R$, which corresponds to the slump flow regime of interest in this paper. The three-dimensional flow problem simplifies then to a one-dimensional equation. The yielding criterion becomes monodimensional and flow simply stops when shear stress in the sample becomes equal or lower than the yield stress $\tau_{c}$. It was shown in [3] that the final shape can then be computed from:

where $h$ is the height, $r$ the radial coordinate, $\rho$ the density and $g$ the gravity. Knowing the sample volume $\Omega$ and neglecting inertia and surface tension [4], the following equation relates the yield stress to the final radius of the sample:

The geometry we consider here is the one suggested in [5] and shown in Fig. 2. The channel width is $200 \mathrm{~mm}$. Its length is higher than $900 \mathrm{~mm}$ and the height of the lateral walls is higher than $150 \mathrm{~mm}$. This geometry allows for a quick and easy measurement of the yield 
1 stress of SCC as there exists an analytical relation between yield stress and final channel flow

2 length $L$ when concrete is not reaching the end of the channel $[5,6]$. This relation writes:

$3 L=\frac{h_{11}}{A}+\frac{i_{i}}{2 A} L N\left(\frac{i_{11}}{i_{y}+2 h_{0}}\right)$

4 Where $h_{0}$ is the thickness of the deposit at $x=0, l_{\mathrm{u}}$ is the width of the channel and $5 \quad A=2 \tau_{\mathrm{r}} / \rho g l_{\|}$.

$7 \quad 3 . \quad$ Numerical methods

8 Table 1 gathers the various numerical methods and their variants used by the researchers 9 involved in the present study. More details on the methods can be found below or in $[2,7$, 10 8].

\section{Computational Fluid Dynamics}

13 Computational Fluid Dynamics (CFD) is the part of fluid mechanics that refers to the use of 14 numerical methods and algorithms to solve and analyze fluid flow problems. Many researchers have carried out simulations of concrete flow within the framework of CFD [2,

16 8]. Simulations have mostly been used to model the flow of fresh concrete during testing 17 including both standard test methods and viscometers. However, a few examples of 18 computational modeling of mixing and full-scale castings can also be found $[2,8]$.

\subsection{Governing equations}

21 The solution to a concrete flow problem involves the computation of properties such as 22 velocity, pressure, density and temperature as functions of space and time from conservation of momentum, mass, and energy. The variation in temperatures can often be 
1 neglected though, as it is for these two benchmark flows. It can be noted that concrete,

2 within this frame, is often assumed to be incompressible.

3

4 4.2. Behavior law

5 When using CFD for concrete, the constitutive equation allowing for the description of the

6 fresh material properties is usually derived from the generalized Newtonian model $[9,10]$. It

7 can either take the form of the Bingham model or of the Herschel Buckley model, which

8 both involves the input of a yield stress that must be exceeded for flow to occur. Examples

9 of more sophisticated material models of thixotropic nature can also be used as presented in

10 [11-13]. From a numerical point of view, implementing a pure yield stress fluid model (i.e.

11 perfect elasto-visco-plastic behavior) is at the origin of many numerical issues. Indeed, in

12 non-flowing zones or in plug-flow zones, the material average strain rate is zero and the

13 apparent local viscosity shall then be infinite and lead to computational divergence.

14 Therefore, approximations based on a smoothing of constitutive relationships often replace

15 the exact behavior laws [14-16]. Using these approximations, the apparent local viscosity is

16 often capped by a maximum value below a critical strain rate. The drawback from such

17 approximations is that the simulated material never really stops flowing. It becomes

18 therefore sometimes necessary to define a flow stop criterion, which allows for the

19 definition of the final shape of the free surface of the material.

21 4.3. Specificities of each team

22 Volume of fluid method - DTU / IFSTTAR / CBI / BAM

23 The volume of fluid method is based on the donor-acceptor approach first introduced in

24 [17]. In a first step, the algorithm computes the volumetric fluxes by geometrically 
1 reconstructing the interface using the values of the fluid fraction function at the previous

2 time step in and around a given control volume. In the second step, the Navier-Stokes

3 equations are solved to compute the velocity field. The fluid is then advected accordingly

4 and the local fluid fraction function is updated. The discretization of the Navier-Stokes

5 equations is carried out with the Finite Volume Method (FVM) in order to obtain the velocity

6 field. In addition, the Volume Of Fluid (VOF) method [17] is used to advect the fluid. In a first

7 step, the VOF algorithm geometrically reconstructs the interface using the values of the fluid

8 fraction function at the previous time step in and around a given control volume. In the

9 second step, the fluid is then advected accordingly by updating the fluid fraction function

10 based on the reconstructed interface and the previously obtained velocity field. The codes

11 used to solve the flow are FLOW3D ${ }^{\circledR}$ for IFSTTAR and DTU, OPENFOAM for CBI and FLUENT ${ }^{\circledR}$

12 for BAM. The models developed here mostly differ by the approximations used to soften the

13 constitutive laws as described above.

14 Particle finite element method - Politecnico di Milano

15 In the approach developed by the group at Politecnico di Milano $[18,19]$, the governing 16 equations are discretized according to the Lagrangian approach, which, by tracking the 17 motion of the material particles, is able to automatically capture the evolution of the free 18 surface configuration, without any a-priori imposed limit to the description of the fluid 19 movement. The FEM mesh remains therefore attached to the material nodes, and a re20 meshing may be necessary to remedy the resulting severe mesh distortion. The constitutive 21 law is the Papanastasiou exponential approximation of a Bingham fluid [16].

\section{Distinct element methods}


1 Discrete numerical simulations of granular materials or Distinct Elements Methods (DEM)

2 give access to mesostructure or even microstructure at the scale of the grains and their

3 contacts using various methods such as the ones described in [2]. These approaches provide

4 therefore an opportunity to study both the flow of the concrete mixture as a whole and the

5 displacement of its individual components. The concrete mixture is modeled by a large

6 number of particles, which are interacting through contact laws. The definition of these

7 contact laws allows for the creation of a virtual concrete mimicking the behavior of the real

8 material.

\section{$10 \quad$ 5.1. Governing equations}

11 In DEM, calculations alternate between the application of Newton's Second Law with

12 respect to the motion of particles and the force-displacement law at the contact points

13 between particles [20-22]. The contact force vector can be divided into normal and shear

14 components with respect to the contact plane. The force-displacement law relates these two

15 force components to the corresponding components of the relative displacement [20-22].

16 The computation time is directly proportional to the number of particles and increases

17 therefore with decreasing particle size for a given material volume in the simulation.

19 5.3. Specificities of each team

\section{IAB Weimar}

21 The IAB Weimar (former IFF Weimar) developed a user defined DEM contact model both in

22 PFC 3D and in EDEM for the representation of fresh concrete. The approach is based on

23 three main aspects: local shear rates, two-layer particle representation and shear angle 
1 dependent normal force. When two particles are in contact, the distance of their centers of

2 mass and their relative velocities are used to compute an approximated local shear rate.

3 Using this shear rate and an approximated contact area, based on the particle sizes and the

4 amount of contacts, the contact force is computed based on the Bingham model and its

5 related parameters: yield stress and plastic viscosity. Using the standard parameters for the

6 Bingham model is easing the calibration of the DEM for a given fresh concrete and also

7 enables a particle size independency of the parameters.

8 In the IAB Weimer approach, the particles are represented as two-layer particles with an

9 inner core representing the real coarse aggregate and an outer core representing the

10 surrounding mortar layer. The outer layer is obviously softer than the inner core and the

11 Bingham model described above is only applied when the outer cores are in contact. As soon

12 as the inner cores get in contact, a normal friction model is applied. The two-layer approach

13 decreases significantly the amount of particles to be computed in comparison to a mortar

14 representation by smaller particles. The computation time is therefore reduced to the same

15 extent.

16 Finally, to reach a smoother movement of the particles and improve the representation of

17 the mortar layer in the outer core, a shear angle dependent normal force is also

18 implemented in the case of a layer contact. This results in a reduction of the additional

19 overlap force in normal direction, depending on the tangential degree of the relative particle

20 movement.

22 TU DRESDEN

23 The interaction laws for the normal and tangential direction used by the team at TU Dresden

24 are shown in Figure 3. They consist of the basic rheological elements spring, dashpot, and 
1 slider, which respectively represent the elastic, viscous and friction components of the

2 particles interaction. A contact element enables the definition of the strength of the contact,

3 the simulation of the loss of an old interaction due to the reaching of a certain distance

4 between two particles, and the formation of a new interaction [22].

5 The force-displacement curve in tension mode is defined for small deformations by a very

6 steep ascending branch (i.e. there is practically no deformation until a given force value

7 (here "yield force") is reached). After reaching this force level, there is only a slight increase

8 in tensile force up to a defined ultimate force (called bond strength) and then a linear

9 decrease to zero in a kind of softening regime. When the tensile force becomes zero,

10 particles lose contact. It can be kept in mind that bond strength is the main parameter

11 defining the interaction of neighboring particles. The bond strength value corresponding to a

12 fresh SCC with yield stress equal to 50 Pa was computed using the methodology presented in $13[23]$.

14 Concrete was modeled by particles of different sizes in such a way that a realistic grading curve of aggregates could be represented with good approximation. The particles were first randomly distributed over a given volume and then compacted under the effect of gravity.

\section{Lattice Boltzmann methods}

19 The Lattice Boltzmann Method (LBM) used at DTU originated around the end of 1980s when cellular automata began to be applied to the field of fluid dynamics. Originally, researchers

21 dealt with lattice gas cellular automata and, only in the beginning of 1990s, LBM was developed and applied to the field of fluid dynamics, see e.g. $[24,25]$ for an overview of the

23 history of the method. LBM, contrary to the aforementioned traditional methods 24 formulating the problem by means of macroscopic quantities such as macroscopic velocity 
1 or pressure fields, is based on Boltzmann equations and thus on the theory of ideal gases. It

2 defines the problem in terms of particle distribution functions, which can be seen as clouds

3 of microscopic particles, e.g. molecules, chaotically moving in space. Space is commonly

4 discretized into square (2D domain) or cubic Eulerian cells forming a fixed Cartesian grid (see

5 Fig. 4)). Eulerian nodes are placed into the centre of each cell and a lattice is formed by connecting the nearest Eulerian nodes. The movement of the microscopic particles is restricted onto the lattice directions, which results in a discretization of continuous particle distribution functions into only a few particle distributions associated with lattice velocities (Fig. 4)).

10 The spacing of nodes, the reference density of the fluid and the length of the time step are usually set to unity for the most simple and common lattice types and the simulated problem is scaled accordingly. Therefore, all quantities are assumed to be dimensionless in this Section. All the equations presented in this section and their derivations can be found e.g. in [26] and references herein. An evolution of the particle distributions in space and time is expressed by the lattice Boltzmann equation, which consists in:

A streaming part, which represents propagation of particle distributions from one node to another along the lattice links. The lattice velocities are chosen in such a way that a particle distribution streams from one node to another during one unit time step.

A forcing term accounting for a modification of particle distributions due to the effect of an external force, see e.g. [27].

A collision part, which mimics real collisions between particles. The collisions are difficult to express explicitly and, therefore, an approximation in the form of a collision operator was introduced into the LBM. The so-called BGK operator, see e.g. [24], linearly deforming the actual particle distributions towards an equilibrium state, is one of the most 
1 widely used collision operators. The equilibrium state is represented by equilibrium particle

2 distributions, which are derived from the Maxwell-Boltzmann equation. The local densities

3 and velocities of the fluid are computed as the first two moments of the particle

4 distributions. The rate of the deformation of the particle distributions towards the

5 equilibrium state is introduced by a relaxation time computed from local macroscopic

6 apparent viscosity (note that kinematic and dynamic viscosity are equal due to the

7 dimensionless form). For non-Newtonian fluids, viscosity is a function of the shear rate and

8 stress deviator tensor, which can be approximated from local off-equilibrium parts of

9 particle distributions [28].

10 Due to the kinematic nature of the LBM and to accuracy and stability issues for high values

11 of relaxation times, behavior of the Bingham plastic cannot be modeled exactly. Recently, a

12 lot of effort has been given into investigations of modeling of Bingham rheology by the LBM,

13 see e.g. [29-32]. Here, the Bingham rheology is approximated by essentially a tri-viscosity

14 fluid, for which we consider that flow has stopped when relaxation time has reached a

15 critical value in a given fraction of the computation cell.

16 We used the Mass Tracking Algorithm [33] to model the free surface. The adopted mass-

17 tracking algorithm is applied directly at the level of the LBM, so the algorithm mimics the

18 free surface by modifying the particle distributions. Lattice cells are differentiated into fluid,

19 gas and interface cells. Gas cells do not contain any particle distributions and thus carry no

20 information, i.e. the LBM is not applied in the gas cells. Fluid cells behave as ordinary LBM

21 cells. Interface cells form a boundary layer between gas cells and fluid cells. Interface cells

22 are treated in the same manner as fluid cells with additional information, namely a local

23 mass of the fluid in the cell. An exchange of mass between cells is calculated based on

24 streamed-out and streamed-in particle distributions. The interface cell becomes a fluid cell 
1 when the mass reaches its density and, vice versa, the interface cell becomes a gas cell when

2 the mass drops down to zero. Particle distributions that would be streamed from gas cells to

3 interface cells do not exist. Therefore, they are reconstructed from particle distributions

4 streamed from interface cells to gas cells based on mass, momentum and pressure

5 equilibriums. Hence, the MTA conserves mass precisely. In the presented benchmarks, we used D3Q15 lattice - a three dimensional lattice with 15 lattice velocities - and spacing of

7 lattice nodes equal to $5 \mathrm{~mm}$.

\section{7. Comparison between numerical predictions}

11 We only focus here on the final shape of the studied concrete volume when flow stops. This 12 information is indeed of first order importance when one is interested in checking if a casting process leads to the proper filling of a given mold or formwork.

\subsection{Slump flow}

We plot in Fig. 5 the various numerical predictions for the benchmark slump flow test studied here. It can first be kept in mind that the approximate analytical solution plotted in

Fig. 5, which predicts a slump flow diameter of $60 \mathrm{~cm}$, is not correct on the symmetry axis.

This solution, to be kept analytical and simple, is indeed based on a scalar plasticity criterion, which only involves shear stress [3]. On this symmetry axis, the stress tensor turns, however,

21 into a purely diagonal matrix, typical of an extensional or elongational flow and the plasticity criterion shall involve the three terms of the diagonal [34]. The shear stress being equal to zero on the axis forces the surface slope of the sample locally to be equal to zero, which is 
1 predicted by most numerical techniques shown in Fig. 5 but not by the approximate

2 analytical solution.

3 It can moreover be noted that CFD methods and LBM, without any fitting, give very similar

4 results and predict a slump flow diameter of average $59.1 \mathrm{~cm}$. The relative standard

5 deviation between these predictions is $1.8 \%$. The DEM methods parameters and particle

6 interaction laws of TU Dresden are fitted on this first benchmark test. After this fitting phase,

7 all methods predict very similar slump flow diameters with an average value of $58.8 \mathrm{~cm}$ and

8 a relative standard deviation of $2.29 \%$.

\subsection{Channel flow}

11 We plot in Fig. 6 the numerical predictions of the channel flow for the various numerical methods presented above. It can be reminded that the exact same input parameters and numerical method as in the previous section are used here. All methods predict very similar profiles at flow stoppage with an average flow length value of $69.7 \mathrm{~cm}$ and a relative standard deviation of $2.95 \%$. The results in Fig. 6, obtained with very different techniques and input parameters, show that these numerical methods are all able to predict concrete flow and the conditions, under which it stops.

\section{Conclusions}

This paper focused on demonstrating that numerical simulations of concrete flow are now

21 fully able to predict accurately concrete flows and that these scientific and engineering tools are ready to be used widely for either academic or industrial purposes.

23 First, we defined two benchmark flows readily usable by anyone calibrating a numerical tool 24 for concrete flow prediction. Such benchmark flows shall allow anyone to check the validity 
1 of their computational tools regardless of the numerical methods and parameters they

2 choose. Second, we compared numerical predictions for these two benchmark flows

3 obtained by various research teams around the world using various numerical techniques.

4 Our results showed that all numerical techniques compared here give very similar results

5 suggesting that numerical simulations of concrete flow have reached a technology readiness

6 level allowing them to move from the lab to the industrial practice.

REFERENCES

9 [1] H. Mori, Y. Tanigawa, Simulation methods for fluidity of fresh Concrete, Memoirs of the

10 School of Engineering, Nagoya University 44 (1992) 71-133.

11 [2] SIMULATION OF FRESH CONCRETE FLOW, 147 Pages, Published: April 2014, Imprint:

12 SPRINGER, ISBN 978-94-017-8883-0

13 [3] Roussel, N., Coussot, P., "Fifty-cent rheometer" for yield stress measurements: from

14 slump to spreading flow, Journal of Rheology, Vol. 49 (2005), º3, pp. 705-718, 2005.

15 [4] N. Roussel, C. Stefani, R. Leroy, From mini cone test to Abrams cone test : measurement 16 of cement based materials yield stress using slump tests, Cem. Concr. Res. 35 (5) (2005) 81717822.

18 [5] Roussel N, The LCPC BOX: a cheap and simple technique for yield stress measurements of 19 SCC, Materials and Structures, 40(9) (2007) 889-896.

20 [6] Nguyen, T.L.H., Roussel, N. Coussot, P., Correlation between L-box test and rheological 21 parameters of a homogeneous yield stress fluid, Cem. Conc. Res., Vol. 36 (2006), N. 10, pp, 1789-1796. 
1 [7] Mechtcherine, V.; Gram, A.; Krenzer, K.; Schwabe, J-H. ; Shyshko, S.; Roussel, N.

2 Simulation of fresh concrete flow using Discrete Element Method (DEM): theory and

3 applications. Materials and Structures, Volume: 47 Issue: 4 Pages: 615-630, 2014.

4 [8] Roussel, N. ; Geiker, M.R. ; Dufour, F. ; Thrane, L.N. ; Szabo, P., Computational modeling 5 of concrete flow: General overview, Cement and Concrete Research, Volume: 37 Issue: 9 6 Pages: 1298-1307, 2007.

7 [9] H.P. Langtangen, Computational Partial Differential Equations, Numerical Methods and 8 Diffpack Programming, Lecture Notes in Computational Sci-ence and Engineering, Springer9 Verlag, Berlin, 1999.

10 [10] R.I. Tanner, K. Walters, Rheology: An Historical Perspective, Elsevier Science B.V., $11 \quad$ Netherlands, 1998.

12 [11] N. Roussel, A thixotropy model for fresh fluid concretes: theory, validation and 13 applications, Cem. Concr. Res. 36 (2006) 1797-1806.

14 [12] J.E. Wallevik, Rheological properties of cement paste: thixotropic behavior and 15 structural breakdown, Cem. Concr. Res. 39 (2009) 14-29.

16 [13] N. Roussel, Steady and transient flow behaviour of fresh cement pastes, Cem. Concr. 17 Res. 35 (2005) 1656-1664.

18 [14] E. O'Donovan, R. Tanner, Numerical study of the Bingham squeeze film problem, Journal 19 of Non-Newtonian Fluid Mechanics 15 (1984) 75-83.

20 [15] G. Lipscomb, M. Denn, Flow of a Bingham fluid in complex geometries, Journal of Non$21 \quad$ Newtonian Fluid Mechanics, 14 (1984) 337-346.

22 [16] T.C. Papanastasiou, Flows of materials with yield, Journal of Rheology, 31 (1987) 38523404. 
1 [17] Hirt, C.W., Nichols, B.D., "Volume of fluid (VOF) method for the dynamics of free

2 boundaries". Journal of Computational Physics 39 (1) (1981) pp. 201-225.

3 [18] M. Cremonesi, A. Frangi, U. Perego, A Lagrangian finite element approach for the 4 analysis of fluid-structure interaction problems, International Journal for Numerical 5 Methods in Engineering, Vol. 84, pp. 610-630, 2010, doi:10.1002/nme.2911.

6 [19] Cremonesi, M., Ferrara, L., Frangi, A., Perego, U.: "Simulation of the flow of fresh 7 cement suspensions by a Lagrangian Finite Element approach", Journal of Non-Newtonian $8 \quad$ Fluid Mechanics, vol. 165, November 2010, pp. 1555-1563.

9 [20] Y. Kishino, Powders and Grains 2001, Proceedings of the fourth interntional conference 10 on micromechnics of granular media, Sendaï, Japan, 21-25 May 2001, A.A. Balkema 11 Publishers.

12 [21] Shyshko, S., Mechtcherine, V.: Simulating the workability of fresh concrete In: 13 Schlangen, E., De Schutter, G. (eds.) Proceedings of the International RILEM Symposium of 14 Concrete Modelling - CONMOD '08, Delft, the Netherlands, 26-28 May 2008, pp. 173-181.

RILEM Publications, Bagneux (2008)

[22] Shyshko, S., Mechtcherine, V.: Developing a Discrete Element Model for simulating fresh concrete: Experimental investigation and modelling of interactions between discrete aggregate particles with fine mortar between them. Construction and Building Materials 47 (2013) 601-615.

[23] V. Mechtcherine, S. Shyshko: Simulating the behaviour of fresh concrete with the Distinct Element Method - Deriving model parameters related to the yield stress, Cement and Concrete Composites, Volume 55, January 2015, Pages 81-90.

[24] S. Chen, G.D. Doolen. Lattice Boltzmann method for fluid flows. Annual Review of Fluid Mechanics, 30(1):329-364, Jan. 1998. 
1 [25] C.K. Aidun, J.R. Clausen. Lattice-Boltzmann Method for Complex Flows. Annual Review

2 of Fluid Mechanics, 42(1):439-472, Jan. 2010.

3 [26] J. Latt. Hydrodynamic limit of lattice Boltzmann equations. PhD thesis, Universite de

4 Geneve, 2007.

5 [27] A. Mohamad, A. Kuzmin. A critical evaluation of force term in lattice Boltzmann method,

6 natural convection problem. International Journal of Heat and Mass Transfer, 53(5-6):9907 996, Feb. 2010.

8 [28] R. Mei, D. Yu,W. Shyy, L.S. Luo. Force evaluation in the lattice Boltzmann method

9 involving curved geometry. Physical Review E, 65(4):1-14, Apr. 2002.

10 [29] A. Vikhansky. Lattice-Boltzmann method for yield-stress liquids. Journal of Non11 Newtonian Fluid Mechanics, 155(3):95-100, Dec. 2008.

12 [30] M. Ohta, T. Nakamura, Y. Yoshida, and Y. Matsukuma. Lattice Boltzmann simulations of 13 viscoplastic fluid flows through complex flow channels. Journal of Non-Newtonian Fluid 14 Mechanics, 166(7-8):404-412, Apr. 2011.

15 [31] Z. Chai, B. Shi, Z. Guo, and F. Rong. Multiple-relaxation-time lattice Boltzmann model for 16 generalized Newtonian fluid flows. Journal of Non-Newtonian Fluid Mechanics, 166(5-6) : 17 332-342, Mar. 2011.

18 [32] C. Leonardi, D. Owen, and Y. Feng. Numerical rheometry of bulk materials using a power 19 law fluid and the lattice Boltzmann method. Journal of Non-Newtonian Fluid Mechanics, 20 166(12-13):628-638, July 2011.

21 [33] C. Körner, M. Thies, T. Hofmann, N. Thürey, and U. Rüde. Lattice Boltzmann Model for 22 Free Surface Flow for Modeling Foaming, Journal of Statistical Physics, 2005, Volume 121, 23 Issue 1-2, pp. 179-196. 
1 [34] Roussel, N., Correlation between yield stress and slump: Comparison between

2 numerical simulations and concrete rheometers results, Materials and Structures, 39(4) pp.

$3501-509,2006$.

4 


\section{Click here to download Table(s): Table 1.docx}

Table 1. Numerical methods and softwares used by the researchers involved in the present study.

\begin{tabular}{|lllll|}
\hline Contact name & Country & Research group & Commercial Software Numerical method \\
Liberato Ferrarra & Italy & Polytechnico di Milano None & CFD - Particle Finite Element method \\
Nicolas Roussel & France & IFSTTAR & FLOW 3D C & CFD - Volume of Fluid method \\
Ksenija Vasilic & Germany & BAM & ANSYS Fluent C & CFD - Volume of Fluid method \\
Annika Gram & Sweden & CBI & OpenFOAM & CFD - Volume of Fluid method \\
Jon Spangerberg & Denmark DTU & FLOW 3D C & CFD - Volume of Fluid method \\
\hline Knut Krenzer & Germany IAB Weimar & PFC 3D C & Distinct Element method \\
Viktor Mechtcherine & Germany TU Dresden & PFC 3D C & Distinct Element method \\
Jan Skocek & Denmark DTU & None & Lattice Boltzman method \\
\hline
\end{tabular}




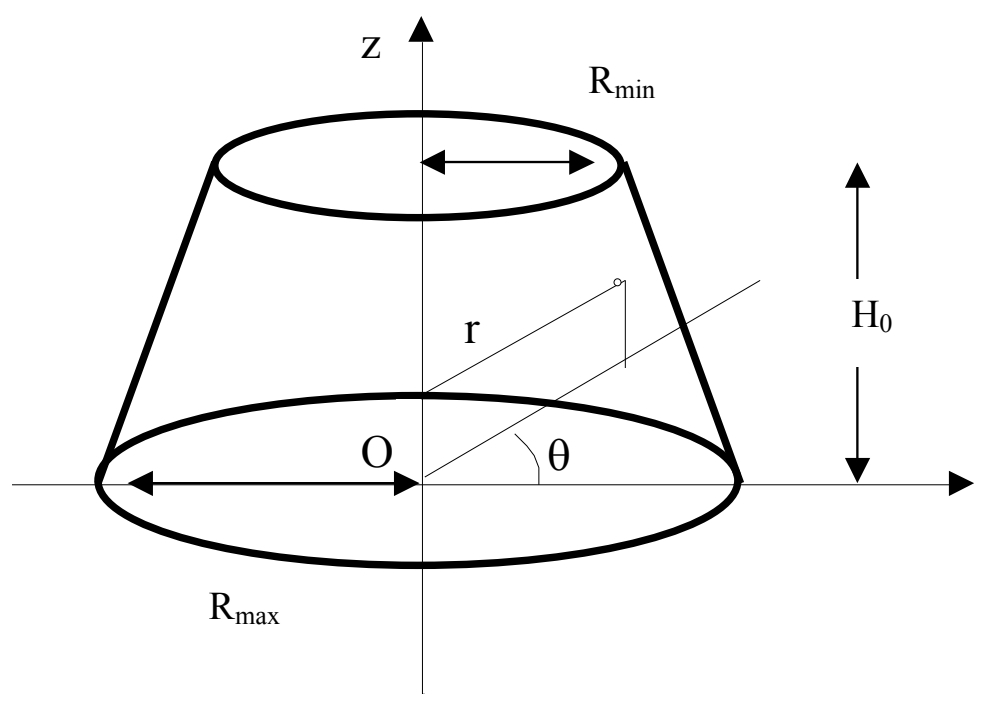

Figure 1. Initial cone shape and cylindrical coordinates. 


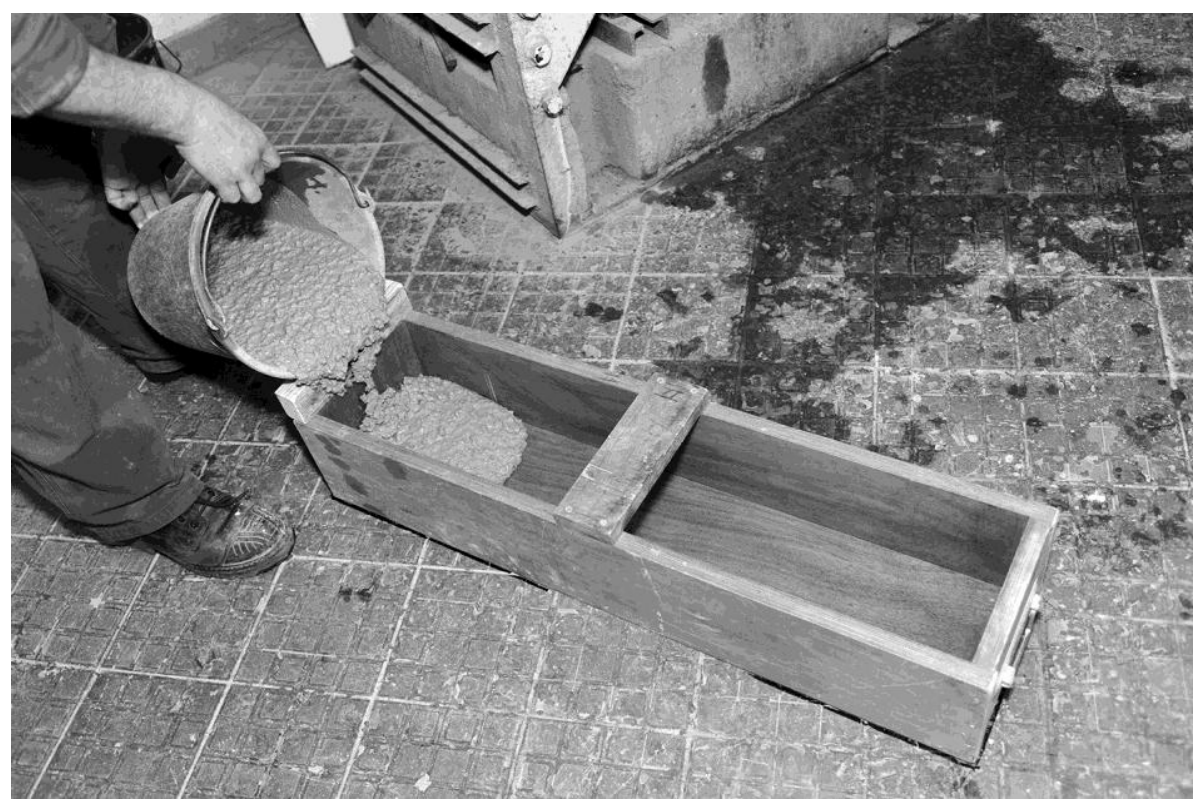

Figure 2. The channel flow and its geometry. 
(a)

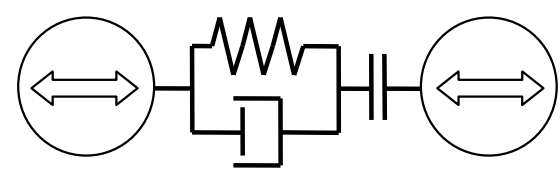

W

Spring

(stiffness)

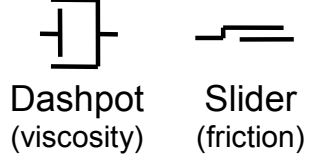

壮

Contact

(force-displacement relation)

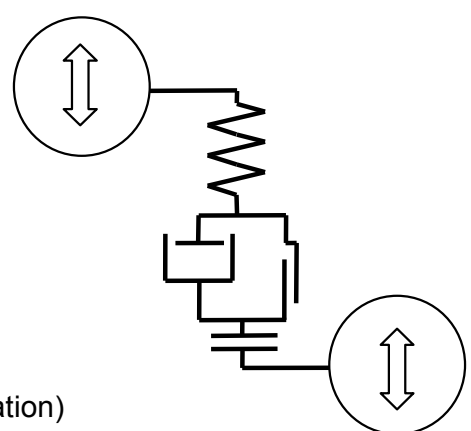

Figure 3. Model for particle interaction: (a) normal direction and (b) tangential direction [22]. 

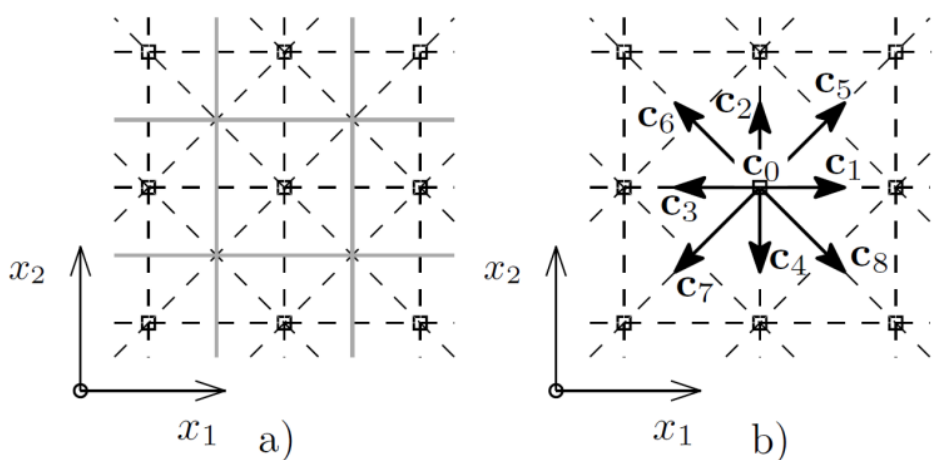

Figure 4. Scheme of so-called D2Q9 lattice for Lattice Boltzmann method. Square marks stand for nodes, grey lines for cell boundaries and dashed lines for the lattice. b) Set of corresponding lattice velocity vectors in a node. 


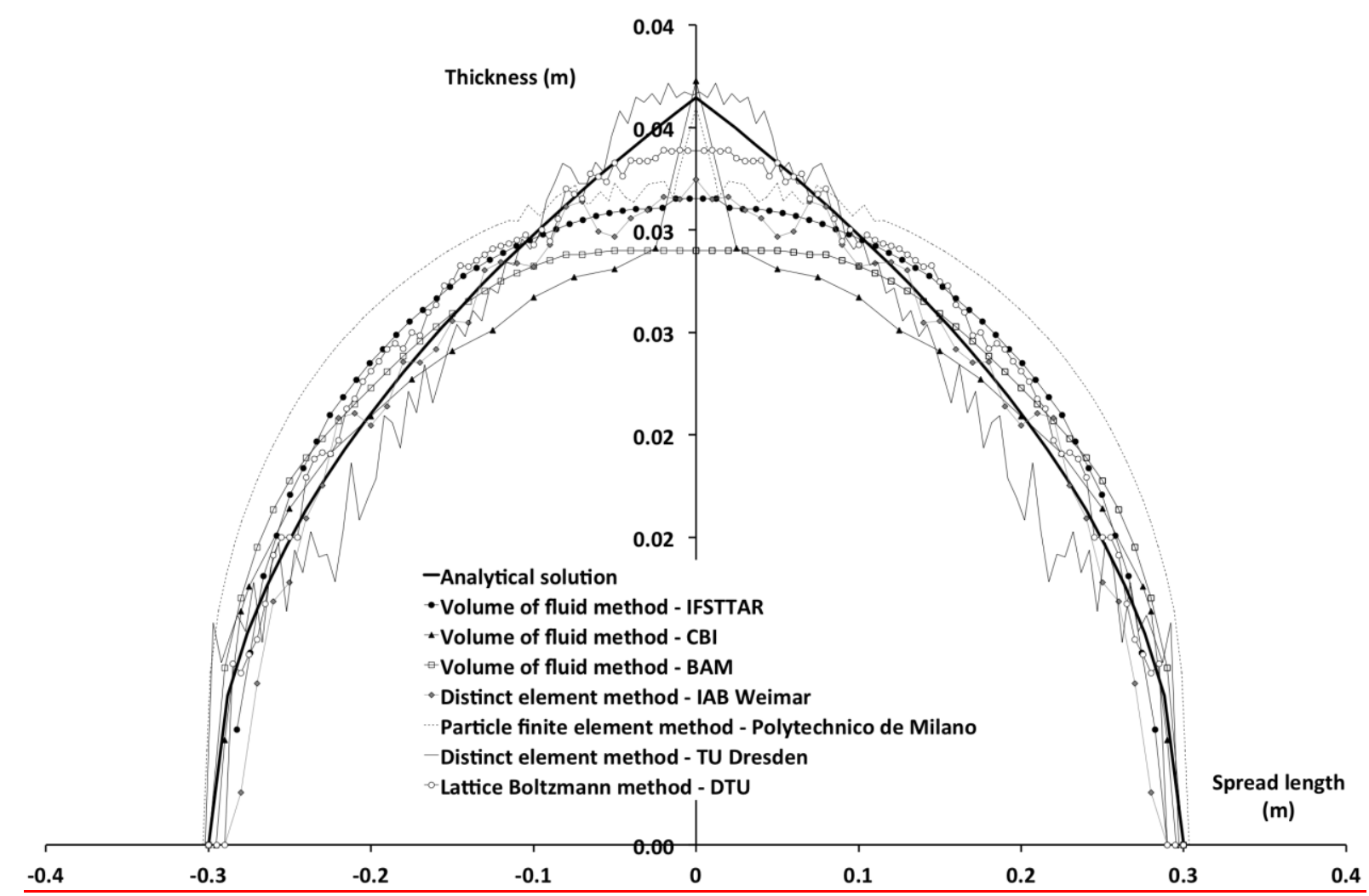

Figure 5. Comparison of the various simulations for the slump flow 


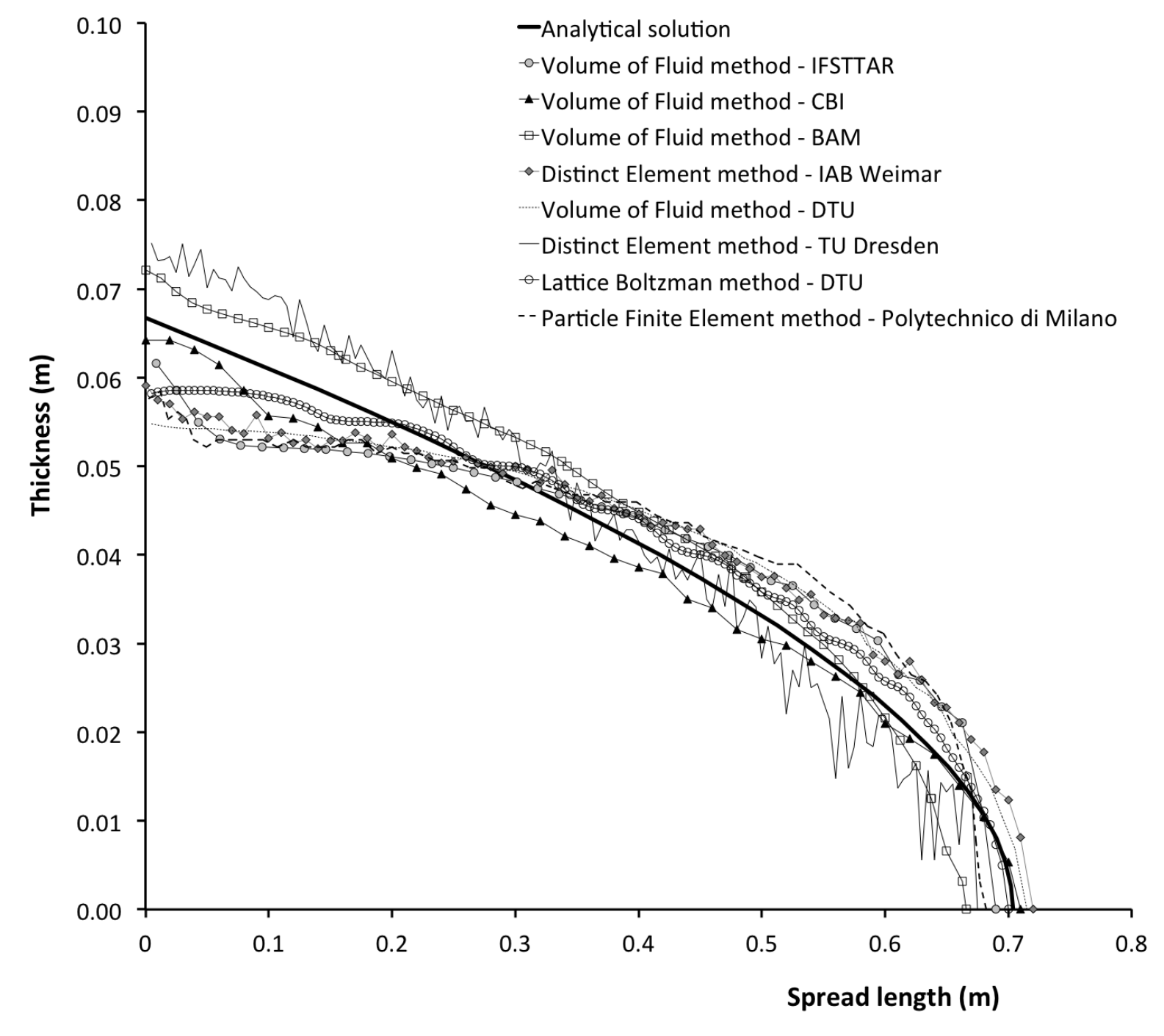

Figure 6. Comparison of the various simulations for the channel flow. 